Journal of Organometallic Chemistry, 388 (1990) 47-55

Elsevier Sequoia S.A., Lausanne - Printed in The Netherlands

JOM 20638

\title{
Synthese von racemischem Acetyl(t-butyl)methylphenylsilan und Acetylmethylphenyl[(trimethylsilyl)methyl]silan: Substrate für stereoselektive mikrobielle Reduktionen
}

\author{
Reinhold Tacke *, Kirsten Fritsche, Andrea Tafel und Frank Wuttke \\ Institut für Anorganische Chemie der Universität Karlsruhe, Engesserstraße, D-7500 Karlsruhe 1 (B.R.D.) \\ (Eingegangen den 8. Dezember 1989)
}

\begin{abstract}
The racemic acetyltriorganylsilanes $\mathrm{MePh}\left(\mathrm{Me}_{3} \mathrm{C}\right) \mathrm{SiC}(\mathrm{O}) \mathrm{Me}(\mathbf{1})$ and $\mathrm{MePh}\left(\mathrm{Me}_{3} \mathrm{Si}-\right.$ $\left.\mathrm{CH}_{2}\right) \mathrm{SiC}(\mathrm{O}) \mathrm{Me}$ (2) were prepared from $\mathrm{MePhSiCl}_{2}$. Compounds 1 and 2 are available by two different three-step syntheses. Method a: $\mathrm{MePhSiCl}_{2} \rightarrow \mathrm{MePh}$ $\left(\mathrm{Me}_{3} \mathrm{C}\right) \mathrm{SiCl}(5) \rightarrow \mathrm{MePh}\left(\mathrm{Me}_{3} \mathrm{C}\right) \mathrm{SiC}(\mathrm{Me}) \mathrm{SCH}_{2} \mathrm{CH}_{2} \mathrm{CH}_{2} \mathrm{~S}(7) \rightarrow 1$ (total yield 36\%); $\mathrm{MePhSiCl}_{2} \rightarrow \mathrm{MePh}\left(\mathrm{Me}_{3} \mathrm{SiCH}_{2}\right) \mathrm{SiCl}(6) \rightarrow \mathrm{MePh}\left(\mathrm{Me}_{3} \mathrm{SiCH}_{2}\right) \mathrm{SiC}(\mathrm{Me}) \mathrm{SCH}_{2} \mathrm{CH}_{2-}$ $\widehat{\mathrm{CH}_{2} \mathrm{~S}}(8) \rightarrow 2$ (total yield 30\%). Method b: $\mathrm{MePhSiCl}_{2} \rightarrow 5 \rightarrow \mathrm{MePh}\left(\mathrm{Me}_{3} \mathrm{C}\right) \mathrm{SiC}-$ $(\mathrm{OMe})=\mathrm{CH}_{2}(9) \rightarrow 1$ (total yield $\left.45 \%\right) ; \mathrm{MePhSiCl}_{2} \rightarrow 6 \rightarrow \mathrm{MePh}\left(\mathrm{Me}_{3} \mathrm{SiCH}_{2}\right) \mathrm{SiC}$ $(\mathrm{OMe})=\mathrm{CH}_{2}(10) \rightarrow 2$ (total yield 33\%). The acetyltriorganylsilanes 1 and 2 and the precursors 5-10 are novel. The silanes 1 and 2 can be used as substrates for enantioselective microbial reductions $\left(\mathrm{R}_{3} \mathrm{SiC}(\mathrm{O}) \mathrm{Me} \rightarrow \mathrm{R}_{3} \mathrm{SiCH}(\mathrm{OH}) \mathrm{Me}\right)$.
\end{abstract}

\section{Zusammenfassung}

Die racemischen Acetyltriorganylsilane $\mathrm{MePh}\left(\mathrm{Me}_{3} \mathrm{C}\right) \mathrm{SiC}(\mathrm{O}) \mathrm{Me}$ (1) und $\mathrm{MePh}\left(\mathrm{Me}_{3} \mathrm{SiCH}_{2}\right) \mathrm{SiC}(\mathrm{O}) \mathrm{Me}(2)$ wurden ausgehend von $\mathrm{MePhSiCl}_{2}$ dargestellt. Beide Verbindungen sind durch zwei verschiedene dreistufige Synthesen zugänglich. Methode a: $\mathrm{MePhSiCl}_{2} \rightarrow \mathrm{MePh}\left(\mathrm{Me}_{3} \mathrm{C}\right) \mathrm{SiCl}(5) \rightarrow \mathrm{MePh}\left(\mathrm{Me}_{3} \mathrm{C}\right) \mathrm{SiC}(\mathrm{Me}) \mathrm{SCH}_{2} \mathrm{CH}_{2}-$ $\widehat{\mathrm{CH}_{2} \mathrm{~S}}(7) \rightarrow 1$ (Gesamtausbeute $36 \%$ ); $\mathrm{MePhSiCl}_{2} \rightarrow \mathrm{MePh}\left(\mathrm{Me}_{3} \mathrm{SiCH}_{2}\right) \mathrm{SiCl}(6) \rightarrow$

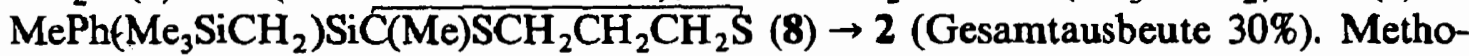
de b: $\mathrm{MePhSiCl}_{2} \rightarrow 5 \rightarrow \mathrm{MePh}\left(\mathrm{Me}_{3} \mathrm{C}\right) \mathrm{SiC}(\mathrm{OMe})=\mathrm{CH}_{2}$ (9) $\rightarrow 1$ (Gesamtausbeute 45\%); $\mathrm{MePhSiCl}_{2} \rightarrow 6 \rightarrow \mathrm{MePh}\left(\mathrm{Me}_{3} \mathrm{SiCH}_{2}\right) \mathrm{SiC}(\mathrm{OMe})=\mathrm{CH}_{2}(10) \rightarrow 2$ (Gesamtausbeute 33\%). Die Acetyltriorganylsilane 1 und 2 sowie die Vorstufen 5-10 wurden erstmalig dargestellt. Die Silane 1 und 2 können als Substrate für enantioselektive mikrobielle Reduktionen eingesetzt werden $\left(\mathrm{R}_{3} \mathrm{SiC}(\mathrm{O}) \mathrm{Me} \rightarrow \mathrm{R}_{3} \mathrm{SiCH}(\mathrm{OH}) \mathrm{Me}\right)$.

\section{Einleitung}

Im Rahmen unserer Arbeiten zur Darstellung optisch aktiver Silane mittels stereoselektiver Biotransformationen (vgl. z.B. Ref. 1-3 (Übersichtsarbeiten) und 
<smiles>[R][Si](C)(C(C)=O)c1ccccc1</smiles>

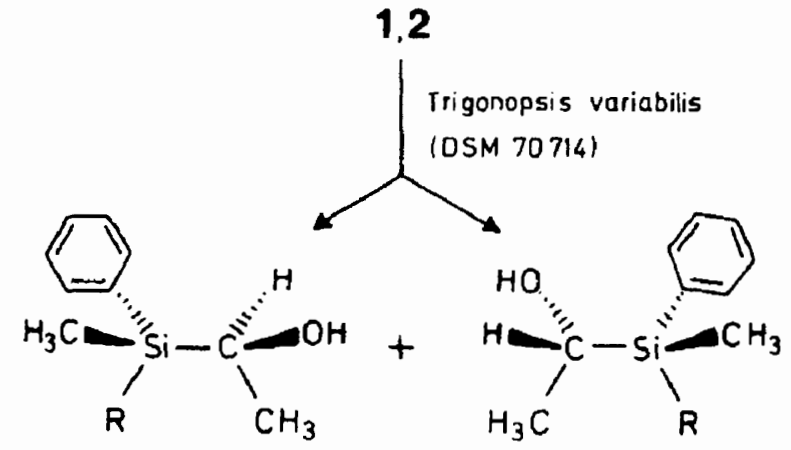

$(S i R, C R)-3$

(SiS,CR)-3

$(S i R, C R)-4$

(SiS, $R$ R) -4

\begin{tabular}{l|l} 
& $\mathrm{R}$ \\
\hline 1,3 & $\mathrm{C}\left(\mathrm{CH}_{3}\right)_{3}$ \\
2,4 & $\mathrm{CH}_{2} \mathrm{Si}_{\left(\mathrm{CH}_{3}\right)_{3}} \quad$ (1,2: Racemate)
\end{tabular}

Schema 1

Ref. 4-7 (Originalarbeiten)) haben wir die racemischen Acetyltriorganylsilane 1 und 2 als Substrate für stereoselek tive mikrobielle Transformationen synthetisiert. Diese Acetylsilane lassen sich an ihren Carbonyl-Gruppen von der Hefe Trigonopsis variabilis (DSM 70714) enantioselektiv ( $R$ )-selektiv) zu den entsprechenden optisch aktiven (1-Hydroxyethyl)silanen ( $\mathrm{Si} R, \mathrm{C} R)-3 /(\mathrm{Si}, \mathrm{C} R)-3$ (96\% ee/96\% ee) und (Si $R, C R)-4 /(\operatorname{Si} S, C R)-4$ (94\% ee $/ 94 \%$ ee) reduzieren (Schema 1; vorläufige Mitteilungen über diese Biotransformationen: Ref. 1-3, 8, 9 (1) und Ref. 2, 3, 9 (2)). Wir berichten hier über die Synthese der racemischen Acetyltriorganylsilane 1 und 2; Details der mit diesen Verbindungen durchgeführten mikrobiellen Transformationen sollen zu gegebener Zeit an anderer Stelle mitgeteilt werden.

\section{Ergebnisse und Diskussion}

Die Darstellung der racemischen Acetyltriorganylsilane 1 und 2 gelang - ausgehend von Dichlormethylphenylsilan - durch jeweils zwei verschiedene dreistufige Synthesen (Schema 2). Die beiden Synthesekonzepte (Methode a und Methode b) basieren auf zwei unterschiedlichen in der Literatur [10-12] beschriebenen Verfahren zur Herstellung von Acetyltriorganylsilanen.

Im jeweils ersten Schritt wurden zunächst durch Umsetzung von Dichlormethylphenylsilan mit t-Butyllithium bzw. [(Trimethylsilyl)methyl]magnesium- 


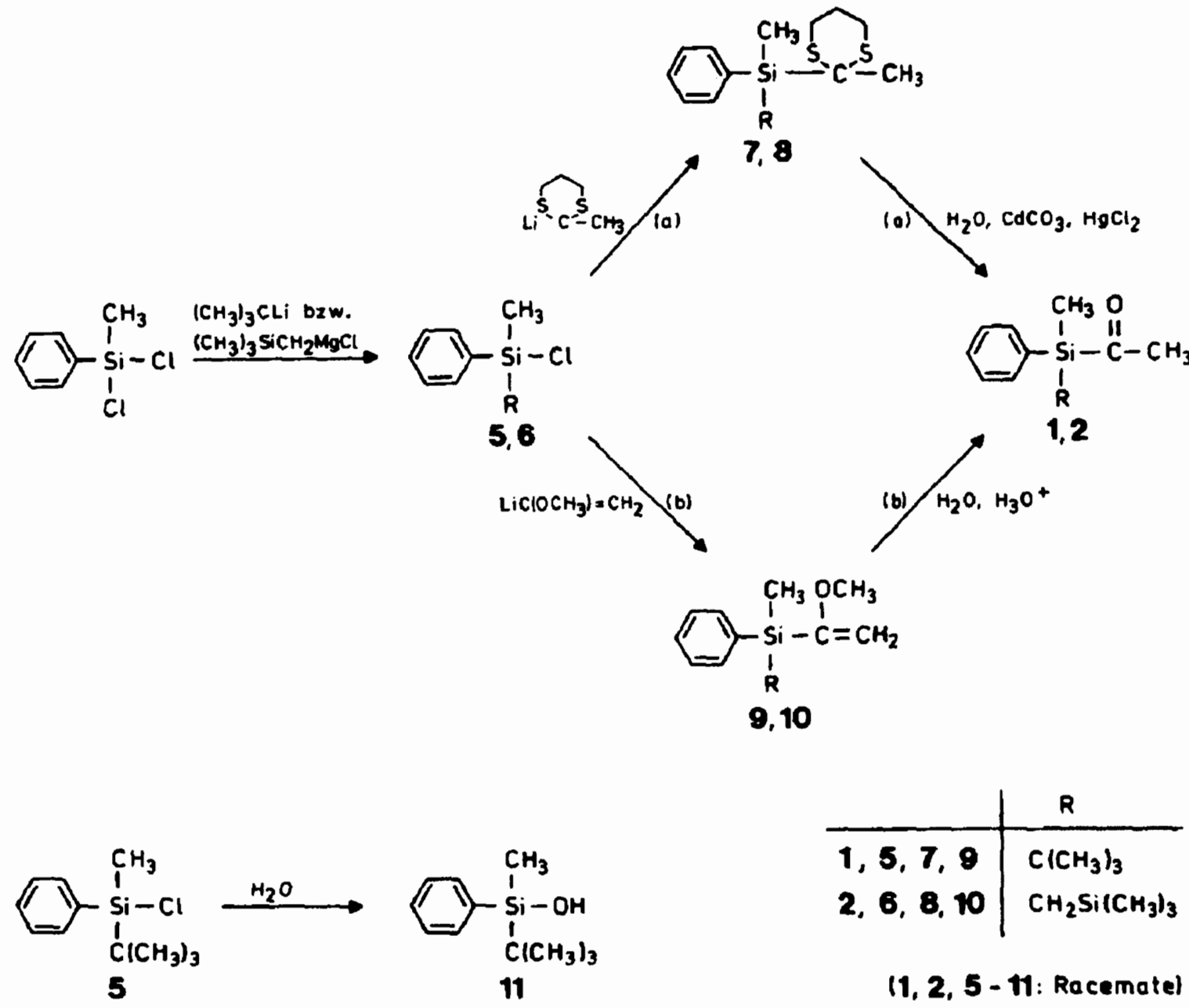

Schema 2

chlorid das entsprechende t-Butylsilan 5 und das [(Trimethylsilyl)methyl]silan 6 dargestellt. Durch deren Umsetzung mit (2-Methyl-1,3-dithiacyclohexan-2-yl)lithium erhielt man die (2-Methyl-1,3-dithiacyclohexan-2-yl)silane 7 und 8, deren nachfolgende Hydrolyse in Gegenwart von Quecksilber(II)chlorid und Cadmiumcarbonat die entsprechenden Acetylsilane 1 bzw. 2 lieferte (Methode a). Die hierbei erzielten Gesamtausbeuten - bezogen auf eingesetztes Dichlormethylphenylsilan - betrugen 36 (1) bzw. 30\% (2).

Die zweite Darstellungsmethode (Methode b) beinhaltete die Umsetzung der Chlorsilane 5 und 6 mit (1-Methoxyvinyl)lithium zu den entsprechenden (1-Methoxyvinyl)silanen 9 bzw. 10 und deren anschließende sauer katalysierte Hydrolyse zu den Acetylsilanen 1 und 2. Diese wurden mit einer Gesamtausbeute - wiederum bezogen auf Dichlormethylphenylsilan - von 45 bzw. 33\% erhalten. Das aus dem (1-Methoxyvinyl)silan 9 erhaltene Acetylsilan 1 wies zunächst geringe Mengen des entsprechenden Silanols 11 (Schema 2) auf, welches sich aber durch Säulenchromatographie an Kieselgel $\left(R_{\mathrm{f}}(\mathbf{1 1})<R_{\mathrm{f}}(\mathbf{1})\right)$ problemlos abtrennen ließ (vgl. hierzu den exp. Teil). Das als Nebenprodukt anfallende Silanol 11 wurde zwecks Vergleich auch durch Hydrolyse des Chlorsilans 5 gezielt hergestellt (Schema 2). 
Das letztgenannte Verfahren (Methode b) zur Darstellung von 1 und 2 ist dem anfangs skizzierten Synthesekonzept (Methode a) nicht nur bezüglich der erzielten Gesamtausbeute, sondern auch aus anderen Gründen überlegen: Die Hydrolyse der (2-Methyl-1,3-dithiacyclohexan-2-yl)silane 7 und 8 erforderte erhebliche Mengen an Quecksilber(II)chlorid und Cadmiumcarbonat (vgl. hierzu den exp. Teil), und die Aufarbeitung der entsprechenden Reaktionsgemische war langwierig und problematisch, wobei sich die vollständige Entfernung quecksilberhaltiger Verunreinigungen aus den Produkten besonders schwierig gestaltete. Derartige SchwermetallVerunreinigungen sind unerwünscht, weil sie aufgrund ihrer biociden Eigenschaften unter Umständen den Ablauf mikrobieller Transformationen von 1 und 2 negativ beeinflussen können.

Die erstmalig dargestellten Acetylsilane 1 und 2, die Zwischenstufen 5-10 sowie das Silanol 11 wurden in ihrer Struktur durch Elementaranalysen sowie durch NMR-spektroskopische $\left({ }^{1} \mathrm{H},{ }^{13} \mathrm{C},{ }^{29} \mathrm{Si}\right)$ und massenspektrometrische Untersuchungen sichergestellt. IR-spektroskopische Untersuchungen von 1 und 2 ergaben typische Werte für die Frequenzen der $\mathrm{CO}-$ Valenzschwingung der $\mathrm{Si}-\mathrm{C}(\mathrm{O})-\mathrm{C}$ Einheiten (beide $1645 \mathrm{~cm}^{-1}$ ), die sich deutlich von den entsprechenden Werten für analoge $\mathrm{C}-\mathrm{C}(\mathrm{O})-\mathrm{C}$-Fragmente unterscheiden (vgl. hierzu Ref. 13). Diese Besonderheiten in den Eigenschaften von Acylsilanen spiegeln sich auch in den entsprechenden ${ }^{13} \mathrm{C}$-NMR-Daten der CO-Gruppe von 1 und 2 wider ( $\delta 244.6$ bzw. 245.3).

Die Acetyltriorganylsilane 1 und 2 können ohne nennenswerte Zersetzungserscheinungen an Kieselgel chromatographiert sowie unter schonenden Bedingungen im Vakuum destilliert werden. Beide Verbindungen lassen sich für mehrere Wochen bei $-20^{\circ} \mathrm{C}$ unter Lichtausschluß weitgehend unzersetzt lagern, längere Lagerzeiten können jedoch zu merklichen Zersetzungserscheinungen führen. Es empfiehlt sich deshalb, die Lagerung auf der Stufe der entsprechenden Vorstufen 7-10 vorzunehmen und dann aus diesen bei Bedarf 1 und $\mathbf{2}$ frisch herzustellen.

\section{Experimentelles}

Alle Reaktionen wurden - wenn nicht anders angegeben - unter einer Schutzgasatmosphäre von trockenem Stickstoff durchgeführt; die verwendeten Lösungsmittel waren wasserfrei. Der Siedebereich des verwendeten Petrolethers betrug $45-70^{\circ} \mathrm{C}$. Das verwendete Kieselgel 60 (Korngröße $0.063-0.200 \mathrm{~mm}$ ) wurde von der Fa. Merck (Best.-Nr. 7734) bezogen. Kugelrohrdestillationen: Kugelrohrverdampfer der Fa. Büchi (Typ GKR-50) mit Glasrohrofen. ${ }^{1} \mathrm{H}-\mathrm{NMR}-S p e k t r e n$ (Lösungsmittel $\mathrm{CDCl}_{3}$, int. Standard $\mathrm{CHCl}_{3}(\delta 7.25 ; 2,5,6,9)$ bzw. TMS ( $\delta 0.00$; übrige Verbindungen)): AM-300-Gerät (300.1 MHz) und WM-400-Gerät (400.13 MHz) der Fa. Bruker. ${ }^{13} \mathrm{C}-\mathrm{NMR}-$ Spektren (Lösungsmittel und int. Standard $\mathrm{CDCl}_{3}(\delta$ 77.05)): AM-300-Gerät (75.47 MHz) und WM-400-Gerät (100.61 MHz) der Fa. Bruker. Die Signalzuordnungen wurden durch DEPT-Experimente unterstützt; die Ergebnisse dieser Experimente sind in den Zuordnungen enthalten. ${ }^{29}$ Si-NMR-Spektren (Lösungsmittel $\mathrm{CDCl}_{3}$, int. Standard TMS ( $\left.\delta 0.00\right)$ ): AC-200-Gerät (39.76 MHz) der Fa. Bruker. IR-Spektren: AccuLab-9-Gerät der Fa. Beckman. Massenspektren

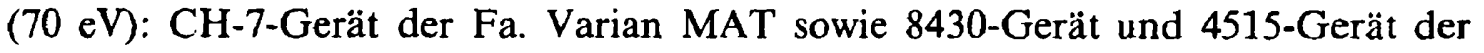
Fa. Finnigan MAT. 


\section{Methode a}

Eine Lösung von $10.87 \mathrm{~g}$ ( $35 \mathrm{mmol}) 7$ in $40 \mathrm{ml}$ Aceton wurde bei $20^{\circ} \mathrm{C}$ unter Rühren innerhalb von $15 \mathrm{~min}$ zu $38.0 \mathrm{~g}$ (140 mmol) $\mathrm{HgCl}_{2}, 12.1 \mathrm{~g}$ (70 mmol) $\mathrm{CdCO}_{3}$ und $6.3 \mathrm{ml}$ Wasser in $240 \mathrm{ml}$ Aceton/Benzol $(1.4 / 1, \mathrm{v} / \mathrm{v})$ getropft. Es wurde $1 \mathrm{~h}$ unter Rückfluß gerührt, auf ca. $10^{\circ} \mathrm{C}$ abgekühlt, filtriert und der Filtrationsrückstand mit Diethylether gründlich gewaschen. Das Filtrat wurde zweimal mit je $250 \mathrm{ml}$ Wasser und zweimal mit je $150 \mathrm{ml}$ einer bei $20^{\circ} \mathrm{C}$ gesättigten wässerigen $\mathrm{NH}_{4} \mathrm{Cl}$-Lösung extrahiert. Die organische Phase wurde über $\mathrm{Na}_{2} \mathrm{SO}_{4}$ getrocknet, unter vermindertem Druck vom Lösungsmittel befreit und der Rückstand durch Kugelrohrdestillation $\left(70-71^{\circ} \mathrm{C} / 0.2\right.$ Torr) gereinigt. Ausb. $5.8 \mathrm{~g}$ (75\%) einer leicht viskosen, farblosen Flüssigkeit. ${ }^{1} \mathrm{H}-\mathrm{NMR}\left(\mathrm{CDCl}_{3}\right): \delta 0.55$ (s, 3H; $\mathrm{SiCH}_{3}$ ), 0.97 (s, 9H; $\left.\mathrm{C}\left(\mathrm{CH}_{3}\right)_{3}\right), 2.29\left(\mathrm{~s}, 3 \mathrm{H} ; \mathrm{C}(\mathrm{O}) \mathrm{CH}_{3}\right), 7.3-7.4$ und 7.5-7.6 (m, 5H; $\left.\mathrm{SiC}_{6} \mathrm{H}_{5}\right) .{ }^{13} \mathrm{C}-\mathrm{NMR}\left(\mathrm{CDCl}_{3}\right): \delta-8.0\left(\mathrm{SiCH}_{3}\right), 17.5\left(\mathrm{C}\left(\mathrm{CH}_{3}\right)_{3}\right), 26.6\left(\mathrm{C}\left(\mathrm{CH}_{3}\right)_{3}\right)$, $38.1\left(\mathrm{C}(\mathrm{O}) \mathrm{CH}_{3}\right), 127.9\left(\mathrm{C}_{m}, \mathrm{SiC}_{6} \mathrm{H}_{5}\right), 129.8\left(\mathrm{C}_{p}, \mathrm{SiC}_{6} \mathrm{H}_{5}\right), 132.6\left(\mathrm{C}_{i}, \mathrm{SiC}_{6} \mathrm{H}_{5}\right), 135.0$ $\left(\mathrm{C}_{o}, \mathrm{SiC}_{6} \mathrm{H}_{5}\right), 244.6\left(\mathrm{SiC}(\mathrm{O}) \mathrm{CH}_{3}\right) .{ }^{29} \mathrm{Si}-\mathrm{NMR}\left(\mathrm{CDCl}_{3}\right): \delta-13.8$. IR $\left(\mathrm{CCl}_{4}\right): 1645$ $\mathrm{cm}^{-1}(\mathrm{C}=\mathrm{O})$. MS: $m / z 220$ (20\%, $\left.M^{+}\right), 135$ (100\%). Gef.: C, 71.0; H, 9.4. $\mathrm{C}_{13} \mathrm{H}_{20} \mathrm{OSi}(220.4)$ ber.: $\mathrm{C}, 70.85 ; \mathrm{H}, 9.15 \%$.

\section{Methode b}

$\mathrm{Zu} 7 \mathrm{ml}$ eines Gemisches aus Aceton und $1 N$ Salzsäure im Volumenverhältnis $4 / 1$ wurden $2.11 \mathrm{~g}(9.0 \mathrm{mmol}) 9$ gegeben. Man rührte $70 \mathrm{~min}$ bei $20^{\circ} \mathrm{C}$ und versetzte dann mit $10 \mathrm{ml}$ Diethylether und $10 \mathrm{ml}$ Wasser. Nach Extraktion und Abtrennung der organischen Phase wurde die wässerige Phase zweimal mit Diethylether extrahiert. Man wusch die vereinigten organischen Extrakte dreimal mit Wasser, trocknete über $\mathrm{Na}_{2} \mathrm{SO}_{4}$ und befreite unter vermindertem Druck vom Lösungsmittel. Das so erhaltene Silan 1 war zu ca. $8 \mathrm{Mol} \%$ ( ${ }^{1} \mathrm{H}-\mathrm{NMR}$ ) durch das Silanol 11 verunreinigt; letzteres wurde durch Säulenchromatographie an Kieselgel 60 mit dem Eluens Diethylether / n-Hexan $(1 / 1.8, \mathrm{v} / \mathrm{v})$ abgetrennt $\left(R_{\mathrm{f}}(11)<R_{\mathrm{f}}(1)\right)$. Das so gereinigte Silan 1 wurde anschließend durch Kugelrohrdestillation $\left(70^{\circ} \mathrm{C} /\right.$ 0.01 Torr) von noch anhaftendem Kieselgel befreit. Ausb. $1.63 \mathrm{~g}$ (82\%). Die spektroskopischen und analytischen Daten des Produktes stimmten mit den unter Methode a angegebenen Werten überein.

\section{Acetylmethylphenyl[(trimethylsilyl)methyl]silan (2)}

\section{Methode a}

Eine Lösung von $3.41 \mathrm{~g}(10 \mathrm{mmol}) 8$ in $10 \mathrm{ml}$ Aceton wurde bei $20^{\circ} \mathrm{C}$ unter Rühren innerhalb von $15 \mathrm{~min}$ zu $13.6 \mathrm{~g}$ (50 mmol) $\mathrm{HgCl}_{2}, 3.45 \mathrm{~g}(20 \mathrm{mmol}) \mathrm{CdCO}_{3}$ und $6.3 \mathrm{ml}$ Wasser in $80 \mathrm{ml}$ Aceton/Benzol (1/4, v/v) getropft. Man rührte $1.5 \mathrm{~h}$ unter Rückfluß, kühlte auf ca. $10^{\circ} \mathrm{C}$ ab, filtrierte und wusch den Filtrationsrückstand gründlich mit kaltem Diethylether. Das Filtrat wurde zweimal mit je $70 \mathrm{ml}$ Wasser und zweimal mit je $50 \mathrm{ml}$ einer bei $20^{\circ} \mathrm{C}$ gesättigten wässerigen $\mathrm{NH}_{4} \mathrm{Cl}$ Lösung gewaschen. Man trocknete die organische Phase über $\mathrm{Na}_{2} \mathrm{SO}_{4}$, entfernte unter vermindertem Druck das Lösungsmittel und reinigte den Rückstand durch Kugelrohrdestillation $\left(70-80^{\circ} \mathrm{C} / 0.01\right.$ Torr). Ausb. $2.40 \mathrm{~g}$ (96\%) einer schwach gelblichen Flüssigkeit. ${ }^{1} \mathrm{H}-\mathrm{NMR}\left(\mathrm{CDCl}_{3}\right): \delta 0.04$ (s, 9H; $\left.\mathrm{Si}\left(\mathrm{CH}_{3}\right)_{3}\right), 0.24$ (s, 
$\left.\boldsymbol{\delta}_{\mathrm{A}}=\boldsymbol{\delta}_{\mathrm{B}}, 2 \mathrm{H} ; \mathrm{SiCH}_{2} \mathrm{Si}\right), 0.54\left(\mathrm{~s}, 3 \mathrm{H} ; \mathrm{SiCH}_{3}\right), 2.19\left(\mathrm{~s}, 3 \mathrm{H} ; \mathrm{C}(\mathrm{O}) \mathrm{CH}_{3}\right), 7.3-7.5(\mathrm{~m}$, $\left.5 \mathrm{H} ; \mathrm{SiC}_{6} \mathrm{H}_{5}\right) \cdot{ }^{13} \mathrm{C}-\mathrm{NMR}\left(\mathrm{CDCl}_{3}\right): \delta-4.5\left(\mathrm{SiCH}_{3}\right),-1.1\left(\mathrm{SiCH} \mathrm{Hi}_{2} \mathrm{Si}\right), 1.2\left(\mathrm{Si}\left(\mathrm{CH}_{3}\right)_{3}\right)$, $35.4\left(\mathrm{C}(\mathrm{O}) \mathrm{CH}_{3}\right), 128.0\left(\mathrm{C}_{m}, \mathrm{SiC}_{6} \mathrm{H}_{5}\right), 129.7\left(\mathrm{C}_{p}, \mathrm{SiC}_{6} \mathrm{H}_{5}\right), 133.9\left(\mathrm{C}_{o}, \mathrm{SiC}_{6} \mathrm{H}_{5}\right), 135.1$ $\left(\mathrm{C}_{i}, \mathrm{SiC}_{6} \mathrm{H}_{5}\right), 245.3\left(\mathrm{SiC}(\mathrm{O}) \mathrm{CH}_{3}\right) .{ }^{29} \mathrm{Si}-\mathrm{NMR}\left(\mathrm{CDCl}_{3}\right): \delta 1.0\left(\mathrm{Si}\left(\mathrm{CH}_{3}\right)_{3}\right),-16.2$ $\left(\mathrm{SiC}(\mathrm{O}) \mathrm{CH}_{3}\right) . \mathrm{IR}(\mathrm{Film}): 1645 \mathrm{~cm}^{-1}(\mathrm{C}=\mathrm{O}) . \mathrm{MS}: m / z 250\left(3 \%, M^{+}\right), 207(100 \%$, $M^{+}-\mathrm{C}(\mathrm{O}) \mathrm{CH}_{3}$ ). Gef.: C, 62.5; H, 9.0; $\mathrm{Si}, 22.6 . \mathrm{C}_{13} \mathrm{H}_{22} \mathrm{OSi}_{2}(250.5)$ ber.: C, 62.34; $\mathrm{H}, 8.85 ; \mathrm{Si}, 22.42 \%$.

\section{Methode $b$}

$\mathrm{Zu} 20 \mathrm{ml}$ eines Gemisches aus Aceton und $1 \mathrm{~N}$ Salzsäure im Volumenverhältnis

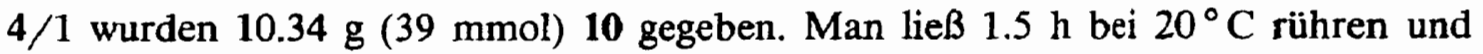
versetzte anschließend mit $16 \mathrm{ml}$ Diethylether und $16 \mathrm{ml}$ Wasser. Nach Extraktion und Abtrennen der organischen Phase extrahierte man die wässerige Phase zweimal mit Diethylether, wusch die vereinigten organischen Extrakte dreimal mit Wasser, trocknete über $\mathrm{MgSO}_{4}$, befreite unter vermindertem Druck vom Lösungsmittel und destillierte den Rückstand im Vakuum über eine Mikro-Vigreuxkolonne. Ausb. $7.8 \mathrm{~g}$ (80\%), Sdp. $80^{\circ} \mathrm{C} / 0.01$ Torr. Die spektroskopischen und analytischen Daten des Produktes stimmten mit den unter Methode a angegebenen Werten überein.

\section{t-Butylchlormethylphenylsilan (5)}

$\mathrm{Zu}$ einer auf $-30^{\circ} \mathrm{C}$ gekühlten Lösung von $47.8 \mathrm{~g}(0.25 \mathrm{~mol})$ Dichlormethylphenylsilan in $200 \mathrm{ml}$ Diethylether wurden unter kräftigem Rühren innerhalb von $1.5 \mathrm{~h} 127.5 \mathrm{ml}(0.255 \mathrm{~mol})$ einer $2 M$ t-Butyllithium-Lösung in n-Pentan getropft. Nach beendeter Zugabe rührte man weitere $2 \mathrm{~h}$ bei $-30^{\circ} \mathrm{C}$, ließ die Lösung innerhalb von $12 \mathrm{~h}$ auf Raumtemperatur erwärmen und erhitzte noch $1 \mathrm{~h}$ unter Rückfluß. Dann wurde der Niederschlag abfiltriert, das Filtrat eingeengt und der Rückstand mit 11 Petrolether versetzt. Man filtrierte erneut, befreite das klare Filtrat unter vermindertem Druck vom Lösungsmittel und destillierte den Rückstand im Vakuum fraktionierend über eine Vigreux-Kolonne. Ausb. $33.7 \mathrm{~g}$ (63\%) einer farblosen Flüssigkeit, Sdp. $45-47^{\circ} \mathrm{C} / 0.1$ Torr. ${ }^{1} \mathrm{H}-\mathrm{NMR}\left(\mathrm{CDCl}_{3}\right): \delta 0.68(\mathrm{~s}, 3 \mathrm{H}$; $\left.\mathrm{SiCH}_{3}\right), 1.00\left(\mathrm{~s}, 9 \mathrm{H} ; \mathrm{C}\left(\mathrm{CH}_{3}\right)_{3}\right), 7.4-7.5$ und 7.6-7.7 (m, 5H; $\left.\mathrm{SiC}_{6} \mathrm{H}_{5}\right) .{ }^{13} \mathrm{C}-\mathrm{NMR}$

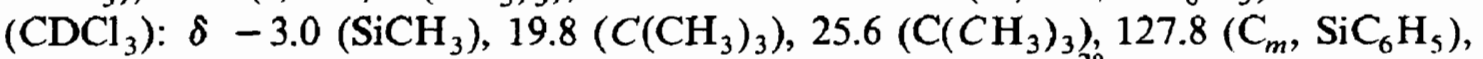
$130.1\left(\mathrm{C}_{p}, \mathrm{SiC}_{6} \mathrm{H}_{5}\right), 133.9\left(\mathrm{C}_{i}, \mathrm{SiC}_{6} \mathrm{H}_{5}\right), 134.3\left(\mathrm{C}_{o}, \mathrm{SiC}_{6} \mathrm{H}_{5}\right) .{ }^{29} \mathrm{Si}-\mathrm{NMR}\left(\mathrm{CDCl}_{3}\right): \delta$ 24.9. MS: $m / z 212\left(9 \%, M^{+}\right), 155\left(100 \%, M^{+}-\mathrm{C}\left(\mathrm{CH}_{3}\right)_{3}\right)$. Gef.: C, 62.6; H, 8.0. $\mathrm{C}_{11} \mathrm{H}_{17} \mathrm{ClSi}(212.8)$ ber.: $\mathrm{C}, 62.09 ; \mathrm{H}, 8.05 \%$.

\section{Chlormethylphenyl[(trimethylsilyl)methyl]silan (6)}

$\mathrm{Zu} 6.1 \mathrm{~g}(0.25 \mathrm{~mol})$ Magnesium (Späne) gab man bei $20^{\circ} \mathrm{C} 40 \mathrm{ml}$ Diethylether und $4 \mathrm{ml}$ von insgesamt $30.7 \mathrm{~g}(0.25 \mathrm{~mol})$ (Chlormethyl)trimethylsilan sowie ein Körnchen lod. Nach dem Anspringen der Reaktion wurde der Rest des (Chlormethyl)trimethylsilans, gelöst in $185 \mathrm{ml}$ Diethylether, unter Rühren so zugetropft, $\mathrm{da} ß$ die Lösung gelinde siedete. Nach beendeter Zugabe ließ man $1 \mathrm{~h}$ unter Rückfluß rühren und tropfte dann das so erhaltene Grignardreagenz bei $20^{\circ} \mathrm{C}$ unter Rühren zu einer Lösung von $47.8 \mathrm{~g}(0.25 \mathrm{~mol})$ Dichlormethylphenylsilan in $175 \mathrm{ml}$ THF. Nach beendeter Zugabe wurde noch 1 h unter Rückfluß gerührt, der Niederschlag abfiltriert, das Filtrat unter vermindertem Druck vom Lösungsmittel befreit und der Rückstand mit $250 \mathrm{ml}$ Petrolether versetzt. Man filtrierte erneut, befreite das Filtrat unter vermindertem Druck vom Lösungsmittel und destillierte 
den Rückstand im Vakuum fraktionierend über eine Vigreux-Kolonne. Ausb. $31.0 \mathrm{~g}$ (51\%) einer farblosen Flüssigkeit, Sdp. $127^{\circ} \mathrm{C} / 15$ Torr. ${ }^{1} \mathrm{H}-\mathrm{NMR}\left(\mathrm{CDCl}_{3}\right): \delta 0.07$ (s, 9H; $\left.\mathrm{Si}\left(\mathrm{CH}_{3}\right)_{3}\right), 0.42$ und 0.47 (AB-System, $J_{\mathrm{AB}} 13.9 \mathrm{~Hz}, 2 \mathrm{H} ; \mathrm{SiCH}_{2} \mathrm{Si}$ ), 0.73 (s, $\left.3 \mathrm{H} ; \mathrm{SiCH}_{3}\right), 7.3-7.7\left(\mathrm{~m}, 5 \mathrm{H} ; \mathrm{SiC}_{6} \mathrm{H}_{5}\right) \cdot{ }^{13} \mathrm{C}-\mathrm{NMR}\left(\mathrm{CDCl}_{3}\right): \delta 1.1\left(\mathrm{Si}\left(\mathrm{CH}_{3}\right)_{3}\right), 3.3$ $\left(\mathrm{SiCH}_{3}\right), 6.5\left(\mathrm{SiCH}_{2} \mathrm{Si}\right), 128.0\left(\mathrm{C}_{m}, \mathrm{SiC}_{6} \mathrm{H}_{5}\right), 130.1\left(\mathrm{C}_{p}, \mathrm{SiC}_{6} \mathrm{H}_{5}\right), 133.1\left(\mathrm{C}_{o}, \mathrm{SiC}_{6} \mathrm{H}_{5}\right)$, $137.5\left(\mathrm{C}_{i}, \mathrm{SiC}_{6} \mathrm{H}_{5}\right)$. MS: m/2 227 (100\%, $\left.M^{+}-\mathrm{CH}_{3}\right)$. Gef.: C, 53.8; H, 8.0; Cl, 14.2; $\mathrm{Si}, 23.2$. $\mathrm{C}_{11} \mathrm{H}_{19} \mathrm{ClSi}_{2}$ (242.9) ber.: C, 54.39; $\mathrm{H}, 7.88 ; \mathrm{Cl}, 14.60 ; \mathrm{Si}, 23.12 \%$.

\section{t-Butylmethyl(2-methyl-1,3-dithiacyclohexan-2-yl)phenylsilan (7)}

$\mathrm{Zu}$ einer auf $-30^{\circ} \mathrm{C}$ gekühlten Lösung von $19.47 \mathrm{~g}(0.145 \mathrm{~mol}) 2-\mathrm{Methyl}-1,3-\mathrm{di}-$ thiacyclohexan in $150 \mathrm{ml}$ THF wurden unter kräftigem Rühren innerhalb von $1 \mathrm{~h} 91$ $\mathrm{ml}$ einer 1.6 $M$ n-Butyllithium-Lösung $(0.146 \mathrm{~mol} \mathrm{BuLi})$ in Hexan getropft. Nach beendeter Zugabe rührte man $60 \mathrm{~min}$ bei $-30^{\circ} \mathrm{C}$, ließ langsam auf $0^{\circ} \mathrm{C}$ erwärmen und tropfte bei dieser Temperatur innerhalb von 70 min eine Lösung von $30.86 \mathrm{~g}$ $(0.145 \mathrm{~mol}) 5 \mathrm{in} 160 \mathrm{ml}$ THF zu dem Reaktionsgemisch. Danach rührte man $3 \mathrm{~h}$ bei $0^{\circ} \mathrm{C}$ und ließ dann innerhalb von $12 \mathrm{~h}$ auf Raumtemperatur erwärmen. Das Lösungsmittel wurde im Rotationsverdampfer unter vermindertem Druck entfernt, der Rückstand in $500 \mathrm{ml}$ Petrolether aufgenommen, die Lösung mit $250 \mathrm{ml} 2 \%$ iger Salzsäure extrahiert und die organische Phase über $\mathrm{Na}_{2} \mathrm{SO}_{4}$ getrocknet. Danach wurde das Lösungsmittel unter vermindertem Druck entfernt und der Rückstand im Vakuum fraktionierend über eine Vigreux-Kolonne destilliert. Ausb. $34.2 \mathrm{~g}$ (76\%) eines ${ }^{1} \mathrm{H}$-NMR-spektroskopisch reinen, gelblichen Öls, Sdp. $138-139^{\circ} \mathrm{C} / 0.1$ Torr. Für analytische Zwecke wurde eine Probe des Destillats einer zusätzlichen Säulenchromatographie an Kieselgel 60 unterzogen (Eluens: Diethylether/n-Hexan $(1 / 4, \mathrm{v} / \mathrm{v}))$. ${ }^{1} \mathrm{H}-\mathrm{NMR}\left(\mathrm{CDCl}_{3}\right): \delta 0.53\left(\mathrm{~s}, 3 \mathrm{H} ; \mathrm{SiCH}_{3}\right), 1.14\left(\mathrm{~s}, 9 \mathrm{H} ; \mathrm{C}\left(\mathrm{CH}_{3}\right)_{3}\right), 1.98$ (s, 3H; $\mathrm{SCCH}_{3}$ ), 1.9-2.1, 2.35-2.5 und 3.1-3.25 (m, 6H; $\left.\mathrm{SCH}_{2} \mathrm{CH}_{2} \mathrm{CH}_{2} \mathrm{~S}\right)$, 7.3-7.4

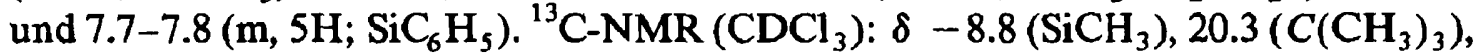

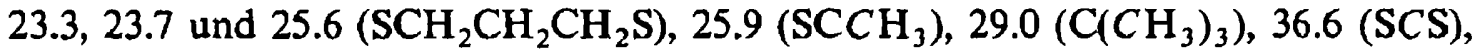
$127.4\left(\mathrm{C}_{m}, \mathrm{SiC}_{6} \mathrm{H}_{5}\right), 129.4\left(\mathrm{C}_{p}, \mathrm{SiC}_{6} \mathrm{H}_{5}\right), 133.8\left(\mathrm{C}_{i}, \mathrm{SiC}_{6} \mathrm{H}_{5}\right), 135.8\left(\mathrm{C}_{o}, \mathrm{SiC}_{6} \mathrm{H}_{5}\right)$. ${ }^{29} \mathrm{Si}-\mathrm{NMR}\left(\mathrm{CDCl}_{3}\right): \delta$ 3.4. MS: $\mathrm{m} / z 310\left(19 \%, \mathrm{M}^{+}\right), 135(100 \%)$. Gef.: C, $61.6 ; \mathrm{H}$, 8.4. $\mathrm{C}_{16} \mathrm{H}_{26} \mathrm{~S}_{2} \mathrm{Si}(310.6)$ ber.: $\mathrm{C}, 61.88 ; \mathrm{H}, 8.44 \%$.

\section{Methyl(2-methyl-1,3-dithiacyclohexan-2-yl)phenyl[(trimethylsilyl)methyl]silan (8)}

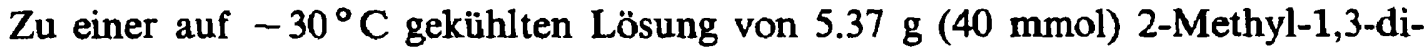
thiacyclohexan in $50 \mathrm{ml}$ THF wurden unter kräftigem Rühren langsam $27 \mathrm{ml}$ einer 1.6 $M$ n-Butyllithium-Lösung (43 mmol BuLi) in Hexan getropft. Nach beendeter Zugabe ließ man $2 \mathrm{~h}$ bei $-30^{\circ} \mathrm{C}$ rühren, überführte das Reaktionsgemisch in einen auf $-20^{\circ} \mathrm{C}$ gekühlten Tropftrichter und tropfte dieses dann unter Rühren langsam zu einer auf $-20^{\circ} \mathrm{C}$ (Temperatur zu Beginn des Zutropfens) gekühlten Lösung von $10.00 \mathrm{~g}$ (41.2 mmol) 6 in $50 \mathrm{ml}$ THF. Während der Zugabe ließ mann von $-20^{\circ} \mathrm{C}$ auf $0^{\circ} \mathrm{C}$ erwärmen. Nach beendeter Zugabe wurde $2 \mathrm{~h}$ bei $0^{\circ} \mathrm{C}$ und weitere $2 \mathrm{~h}$ bei $20^{\circ} \mathrm{C}$ gerührt, das Lösungsmittel unter vermindertem Druck entfernt und der Ruickstand mit $500 \mathrm{ml}$ Petrolether versetzt. Dann extrahierte man vorsichtig mit 2\% iger Salzsäure, trennte die wässerige Lösung $\mathrm{ab}$, wusch die organische Phase mit Wasser neutral, trocknete diese über $\mathrm{Na}_{2} \mathrm{SO}_{4}$, entfernte das Lösungsmittel unter vermindertem Druck und destillierte den Rückstand im Kugelrohrverdampfer (120$140^{\circ} \mathrm{C} / 0.01$ Torr). Das Destillat wurde anschließend einer fraktionierenden Vakuumdestillation über eine kurze Vigreux-Kolonne unterworfen. Ausb. $8.75 \mathrm{~g}$ (62\%) 
einer farblosen viskosen Flüssigkeit, Sdp. $139^{\circ} \mathrm{C} / 0.05$ Torr. ${ }^{1} \mathrm{H}-\mathrm{NMR}\left(\mathrm{CDCl}_{3}\right)$ : $\delta$ -0.08 (s, 9H; $\left.\mathrm{Si}\left(\mathrm{CH}_{3}\right)_{3}\right), 0.35$ und 0.51 (AB-System, $J_{\mathrm{AB}} 14.0 \mathrm{~Hz}, 2 \mathrm{H} ; \mathrm{SiCH}_{2} \mathrm{Si}$ ). $0.59\left(\mathrm{~s}, 3 \mathrm{H} ; \mathrm{SiCH}_{3}\right), 1.81\left(\mathrm{~s}, 3 \mathrm{H} ; \mathrm{CCH}_{3}\right), 1.8-2.1,2.4-2.5$ und $3.0-3.1(\mathrm{~m}, 6 \mathrm{H}$; $\left.\mathrm{SCH}_{2} \mathrm{CH}_{2} \mathrm{CH}_{2} \mathrm{~S}\right), 7.3-7.7\left(\mathrm{~m}, 5 \mathrm{H} ; \mathrm{SiC}_{6} \mathrm{H}_{5}\right) .{ }^{13} \mathrm{C}-\mathrm{NMR}\left(\mathrm{CDCl}_{3}\right): \delta-5.7\left(\mathrm{SiCH}_{3}\right)$, - $0.3\left(\mathrm{SiCH}{ }_{2} \mathrm{Si}\right), 1.4\left(\mathrm{Si}\left(\mathrm{CH}_{3}\right)_{3}\right), 23.3,23.5$ und $25.5\left(\mathrm{SCH}_{2} \mathrm{CH}_{2} \mathrm{CH}_{2} \mathrm{~S}\right), 24.3\left(\mathrm{CCH}_{3}\right)$, 35.5 (SCS), $127.4\left(\mathrm{C}_{m}, \mathrm{SiC}_{6} \mathrm{H}_{5}\right), 129.6\left(\mathrm{C}_{p}, \mathrm{SiC}_{6} \mathrm{H}_{5}\right), 134.5\left(\mathrm{C}_{i}, \mathrm{SiC}_{6} \mathrm{H}_{5}\right), 135.1\left(\mathrm{C}_{o}\right.$, $\left.\mathrm{SiC}_{6} \mathrm{H}_{5}\right)$. MS: $m / z 340\left(26 \%, M^{+}\right), 207\left(100 \%, M^{+}-\mathrm{H}_{3} \mathrm{CCSCH}_{2} \mathrm{CH}_{2} \mathrm{CH}_{2} \mathrm{~S}\right)$. Gef.: C, 55.9; H, 8.1; Si, 16.4. $\mathrm{C}_{16} \mathrm{H}_{28} \mathrm{~S}_{2} \mathrm{Si}_{2}$ (340.7) ber.: C, 56.41; H, 8.28; Si, 16.49\%.

\section{t-Butyl(1-methoxyvinyl)methylphenylsilan (9)}

$\mathrm{Zu}$ einer Lösung von $26.3 \mathrm{ml}\left(0.35 \mathrm{~mol} ; d\left(0^{\circ} \mathrm{C}\right) 0.77 \mathrm{~g} / \mathrm{cm}^{3}\right)$ Methylvinylether in $120 \mathrm{ml}$ THF wurden bei $-78^{\circ} \mathrm{C}$ unter Rühren innerhalb von $90 \mathrm{~min} 176 \mathrm{ml}$ einer 1.6 $M$ t-Butyllithium-Lösung $(0.282 \mathrm{~mol} \mathrm{BuLi})$ in Pentan getropft, wobei sich ein zitronengelber Niederschlag bildete. Nach beendeter Zugabe ließ man innerhalb von $3 \mathrm{~h}$ auf $0^{\circ} \mathrm{C}$ erwärmen (Entfärbung, Auflösung des Niederschlages), kühlte das Reaktionsgemisch auf $-50^{\circ} \mathrm{C}$ ab und tropfte hierzu innerhalb von 60 min unter Rühren $46.8 \mathrm{~g}$ ( $0.22 \mathrm{~mol}) 5$. Nach beendeter Zugabe ließ man innerhalb von $12 \mathrm{~h}$ auf Raumtemperatur erwärmen, extrahierte das Reaktionsgemisch zweimal mit je 100 $\mathrm{ml}$ einer bei $20^{\circ} \mathrm{C}$ gesättigten wässerigen $\mathrm{NH}_{4} \mathrm{Cl}$-Lösung, trennte die organische Phase $a b$, extrahierte die vereinigten wässerigen Phasen zweimal mit je $25 \mathrm{ml}$ n-Pentan, trocknete die vereinigten organischen Extrakte über $\mathrm{K}_{2} \mathrm{CO}_{3}$, befreite unter vermindertem Druck vom Lösungsmittel und destillierte den Rückstand im Vakuum fraktionierend über eine Vigreux-Kolonne. Ausb. $44.8 \mathrm{~g}(87 \%)$ einer farblosen Flüssigkeit, Sdp. $60-62^{\circ} \mathrm{C} / 0.05$ Torr. ${ }^{1} \mathrm{H}-\mathrm{NMR}\left(\mathrm{CDCl}_{3}\right): \delta 0.43(\mathrm{~s}, 3 \mathrm{H}$; $\left.\mathrm{SiCH}_{3}\right), 1.01$ (s, 9H; $\left.\mathrm{C}\left(\mathrm{CH}_{3}\right)_{3}\right), 3.62\left(\mathrm{~s}, 3 \mathrm{H} ; \mathrm{OCH}_{3}\right), 4.47$ und 4.82 (beide d, $J 2.3$ $\left.\mathrm{Hz}, 2 \mathrm{H} ; \mathrm{SiC}=\mathrm{CH}_{2}\right), 7.3-7.4$ und 7.7-7.8 (m, 5H; $\left.\mathrm{SiC}_{6} \mathrm{H}_{5}\right) \cdot{ }^{13} \mathrm{C}-\mathrm{NMR}\left(\mathrm{CDCl}_{3}\right): \delta$ - $7.4\left(\mathrm{SiCH}_{3}\right), 17.3\left(\mathrm{C}\left(\mathrm{CH}_{3}\right)_{3}\right), 27.2\left(\mathrm{C}\left(\mathrm{CH}_{3}\right)_{3}\right), 54.0\left(\mathrm{OCH}_{3}\right), 96.7\left(\mathrm{SiC}=\mathrm{CH}_{2}\right)$, $127.5\left(\mathrm{C}_{m}, \mathrm{SiC}_{6} \mathrm{H}_{5}\right), 129.1\left(\mathrm{C}_{p}, \mathrm{SiC}_{6} \mathrm{H}_{5}\right), 135.2\left(\mathrm{C}_{o}, \mathrm{SiC}_{6} \mathrm{H}_{5}\right), 135.6\left(\mathrm{C}_{i}, \mathrm{SiC}_{6} \mathrm{H}_{5}\right)$, $168.4\left(\mathrm{SiC}=\mathrm{CH}_{2}\right) .{ }^{29} \mathrm{Si}+\mathrm{NMR}\left(\mathrm{CDCl}_{3}\right): \delta-6.4$. MS: $m / z 234\left(4 \%, M^{+}\right), 151$ (100\%). Gef.: C, 71.3; H, 9.7. $\mathrm{C}_{14} \mathrm{H}_{22} \mathrm{OSi}$ (234.4) ber.: C, 71.73; H, 9.46\%.

\section{(1-Methoxyvinyl)methylphenyl[(trimethylsilyl)methyl]silan (10)}

$\mathrm{Zu}$ einer Lösung von $6.2 \mathrm{ml}\left(82 \mathrm{mmol} ; d\left(0^{\circ} \mathrm{C}\right) 0.77 \mathrm{~g} / \mathrm{cm}^{3}\right)$ Methylvinylether in $42 \mathrm{ml}$ THF wurden bei $-78^{\circ} \mathrm{C}$ unter Rühren innerhalb von $30 \mathrm{~min} 52 \mathrm{ml}$ einer 1.6 $M$ t-Butyllithium-Lösung ( $83 \mathrm{mmol} \mathrm{BuLi})$ in Pentan getropft, wobei sich ein zitronengelber Niederschlag bildete. Nach beendeter Zugabe ließ man innerhalb von $3 \mathrm{~h}$ auf $0^{\circ} \mathrm{C}$ erwärmen (Entfärbung, Auflösung des Niederschlages), kühlte das Reaktionsgemisch auf $-50^{\circ} \mathrm{C}$ ab und tropfte hierzu innerhalb von 30 min unter Rühren $12.15 \mathrm{~g}(50 \mathrm{mmol}) 6$. Nach beendeter Zugabe ließ man langsam auf $20^{\circ} \mathrm{C}$ erwärmen, extrahierte das Reaktionsgemisch zweimal mit einer bei $20^{\circ} \mathrm{C}$ gesättigten wässerigen $\mathrm{NH}_{4} \mathrm{Cl}$-Lösung, trennte die organische Phase ab, extrahierte die vereinigten wässerigen Phasen zweimal mit je $25 \mathrm{ml} n$-Pentan, trocknete die vereinigten organischen Extrakte über $\mathrm{K}_{2} \mathrm{CO}_{3}$, befreite unter vermindertem Druck vom Lösungsmittel und destillierte den Rückstand im Vakuum fraktionierend über eine Vigreux-Kolonne. Ausb. $10.8 \mathrm{~g}(82 \%)$ einer farblosen Flüssigkeit, Sdp. $83^{\circ} \mathrm{C} / 0.1$ Torr. ${ }^{1} \mathrm{H}-\mathrm{NMR}\left(\mathrm{CDCl}_{3}\right): \delta 0.01\left(\mathrm{~s}, 9 \mathrm{H} ; \mathrm{Si}\left(\mathrm{CH}_{3}\right)_{3}\right), 0.13$ und 0.25 (AB-System, $J_{\mathrm{AB}}$ $\left.13.8 \mathrm{~Hz}, 2 \mathrm{H} ; \mathrm{SiCH}_{2} \mathrm{Si}\right), 0.46\left(\mathrm{~s}, 3 \mathrm{H} ; \mathrm{SiCH}_{3}\right), 3.55\left(\mathrm{~s}, 3 \mathrm{H} ; \mathrm{OCH}_{3}\right), 4.40$ und 4.72 (beide d, $J 2.0 \mathrm{~Hz}, 2 \mathrm{H}$; $\left.\mathrm{SiC}=\mathrm{CH}_{2}\right), 7.4-7.7\left(\mathrm{~m}, 5 \mathrm{H} ; \mathrm{SiC}_{6} \mathrm{H}_{5}\right) \cdot{ }^{13} \mathrm{C}-\mathrm{NMR}\left(\mathrm{CDCl}_{3}\right): \delta$ 
- $2.9\left(\mathrm{SiCH}_{3}\right), 0.3\left(\mathrm{SiCH}_{2} \mathrm{Si}\right), 1.2\left(\mathrm{Si}\left(\mathrm{CH}_{3}\right)_{3}\right), 54.2\left(\mathrm{OCH}_{3}\right), 95.1\left(\mathrm{SiC}=\mathrm{CH}_{2}\right), 127.7$ $\left(\mathrm{C}_{m}, \mathrm{SiC}_{6} \mathrm{H}_{5}\right), 129.0\left(\mathrm{C}_{p}, \mathrm{SiC}_{6} \mathrm{H}_{5}\right), 134.0\left(\mathrm{C}_{o}, \mathrm{SiC}_{6} \mathrm{H}_{5}\right), 138.2\left(\mathrm{C}_{i}, \mathrm{SiC}_{6} \mathrm{H}_{5}\right), 169.5$ $\left(\mathrm{SiC}=\mathrm{CH}_{2}\right)$. MS: $m / z 249\left(100 \%, M^{+}-\mathrm{CH}_{3}\right)$. Gef.: C, 63.6; H, 9.4; Si, 20.7. $\mathrm{C}_{14} \mathrm{H}_{24} \mathrm{OSi}_{2}$ (264.5) ber.: C, 63.57; H, 9.14; $\mathrm{Si}, 21.24 \%$.

\section{t-Butylmethylphenylsilanol (II)}

$\mathrm{Zu}$ einer Lösung von $600 \mathrm{mg}(2.82 \mathrm{mmol}) 5$ in $20 \mathrm{ml}$ Diethylether wurden bei Raumtemperatur unter Rühren $2 \mathrm{ml}$ Wasser getropft. Man rührte $3 \mathrm{~h}$ bei Raumtemperatur, trennte die organische Phase ab, extrahierte diese zweimal mit je $2 \mathrm{ml}$ einer gesättigten wässerigen $\mathrm{NaHCO}_{3}$-Lösung und trocknete die organische Phase über $\mathrm{Na}_{2} \mathrm{SO}_{4}$. Das Lösungsmittel wurde unter vermindertem Druck entfernt und der Rückstand im Kugelrohrverdampfer destilliert ( $78^{\circ} \mathrm{C} / 0.1$ Torr). Ausb. $510 \mathrm{mg}$ (93\%) einer farblosen, leichtviskosen Flüssigkeit. ${ }^{1} \mathrm{H}-\mathrm{NMR}\left(\mathrm{CDCl}_{3}\right): \delta 0.39(\mathrm{~s}, 3 \mathrm{H}$; $\left.\mathrm{SiCH}_{3}\right), 0.95\left(\mathrm{~s}, 9 \mathrm{H} ; \mathrm{Si}\left(\mathrm{CH}_{3}\right)_{3}\right), 7.3-7.4$ und 7.5-7.6 (m, 5H; $\left.\mathrm{SiC}_{6} \mathrm{H}_{5}\right) .{ }^{13} \mathrm{C}-\mathrm{NMR}$

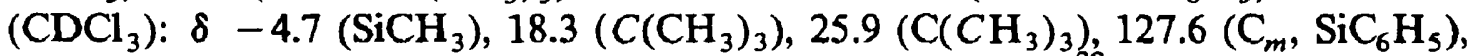
$129.4\left(\mathrm{C}_{p}, \mathrm{SiC}_{6} \mathrm{H}_{5}\right), 134.1\left(\mathrm{C}_{o}, \mathrm{SiC}_{6} \mathrm{H}_{5}\right), 137.0\left(\mathrm{C}_{i}, \mathrm{SiC}_{6} \mathrm{H}_{5}\right) .{ }^{29} \mathrm{Si}-\mathrm{NMR}\left(\mathrm{CDCl}_{3}\right): \delta$ 8.5. IR $\left(\mathrm{CCl}_{4}\right): 3440\left(\mathrm{OH}\right.$, assoziiert), $3665 \mathrm{~cm}^{-1}(\mathrm{OH}$, nicht assoziiert). MS: $m / z$ $194\left(4 \%, M^{+}\right), 137\left(100 \%, M-\mathrm{C}\left(\mathrm{CH}_{3}\right)_{3}\right)$. Gef.: C, 67.6; H, 9.3. $\mathrm{C}_{11} \mathrm{H}_{18} \mathrm{OSi}(194.3)$ ber.: C, 67.98; H, 9.33\%.

\section{Dank}

Wir danken dem Fonds der Chemischen Industrie für die finanzielle Unterstützung dieser Arbeit. Der Bayer AG, Leverkusen und Wuppertal-Elberfeld, gilt unser Dank für großzügige Chemikalienspenden. Der BASF, Ludwigshafen, sei für die Bereitstellung des Methylvinylethers gedankt.

\section{Literatur}

1 R. Tacke und B. Becker, Main Group Met. Chem., 10 (1987) 169.

2 C. Syldatk, A. Stoffregen, A. Brans, K. Fritsche, H. Andree, F. Wagner, H. Hengelsberg, A. Tafel, F. Wuttke, H. Zilch und R. Tacke, in H.W. Blanch und A.M. Klibanov (Hrsg.), Enzyme Engineering 9, Annals of the New York Academy of Sciences, Vol. 542. The New York Academy of Sciences, New York, 1988, S. 330-338.

3 R. Tacke und H. Linoh, in S. Patai und Z. Rappoport (Hrsg.), The Chemistry of Organic Silicon Compounds, Part 2, John Wiley \& Sons Ltd., Chichester, 1989, S. 1143-1206.

4 C. Syldatk, H. Andree, A. Stoffregen, F. Wagner, B. Stumpf, L. Ernst, H. Zilch und R. Tacke, Appl. Microbiol. Biotechnol., 27 (1987) 152.

5 C. Syldatk, A. Stoffregen, F. Wuttke und R. Tacke, Biotechnol. Lett., 10 (1988) 731.

6 K. Fritsche, C. Syldatk, F. Wagner, H. Hengelsberg und R. Tacke, Appl. Microbiol. Biotechnol., 31 (1989) 107.

7 R. Tacke, H. Hengelsberg, H. Zilch und B. Stumpf, J. Organomet. Chem., 379 (1989) 211.

8 R. Tacke, F. Wuttke, H. Zilch, H. Andree, C. Syldatk, F. Wagner, L. Ernst und D. Schomburg, VIth FECHEM Conference on Organometallic Chemistry, Riga 1985, Abstracts, S. 188.

9 R. Tacke, K. Fritsche, H. Hengelsberg, A. Tafel, F. Wuttke, H. Zilch, C. Syldatk, A. Brans und F. Wagner, 1st International Symposium on Separation of Chiral Molecules, Paris 1988, Programme, S. 30-31.

10 D. Seebach, Synthesis, (1969) 17.

11 E.M. Dexheimer und L. Spialter, J. Organomet. Chem., 107 (1976) 229.

12 J.A. Soderquist und G. Ji-Ho Hsu, Organometallics, 1 (1982) 830.

13 A.G. Brook, Adv. Organomet. Chem., 7 (1968) 95. 